DOI: $10.15393 /$ j3.art.2019.6350

UDC 517.38, 517.51

B. Moosavi, H. R. Moradi, M. Shah Hosseini

\title{
FURTHER RESULTS ON JENSEN-TYPE INEQUALITIES
}

\begin{abstract}
In this paper, we establish some Jensen-type inequalities for continuous functions of self-adjoint operators on complex Hilbert spaces. Furthermore, using the Cartesian decomposition of an operator, we improve the known result due to Mond and Pečarić. Some refinements of the Hölder-McCarthy inequality are given as well.
\end{abstract}

Key words: Jensen's inequality, convex function, synchronous (asynchronous) function, self-adjoint operator

2010 Mathematical Subject Classification: Primary 47A63, Secondary 26A51, 26D15, 26B25, 39B62

1. Introduction and Preliminaries. Let $\mathcal{B}(\mathcal{H})$ be the $C^{*}$ - algebra of all bounded linear operators on a Hilbert space $\mathcal{H}$. As customary, we reserve $m, M$ for scalars and $\mathbf{1}_{\mathcal{H}}$ for the identity operator on $\mathcal{H}$. A selfadjoint operator $A$ is said to be positive (written $A \geqslant 0$ ) if $\langle A x, x\rangle \geqslant 0$ holds for all $x \in \mathcal{H}$; also an operator $A$ is said to be strictly positive (denoted by $A>0)$ if $A$ is positive and invertible. If $A$ and $B$ are self-adjoint, we write $B \geqslant A$ in case $B-A \geqslant 0$. The Gelfand map $f(t) \mapsto f(A)$ is an isometrical $*$ - isomorphism between the $C^{*}$ - algebra $C(\operatorname{sp}(A))$ of continuous functions on the spectrum $s p(A)$ of a self-adjoint operator $A$ and the $C^{*}$ - algebra generated by $A$ and the identity operator $\mathbf{1}_{\mathcal{H}}$. If $f, g \in C(s p(A))$, then $f(t) \geqslant g(t)(t \in \operatorname{sp}(A))$ implies that $f(A) \geqslant g(A)$.

For $A, B \in \mathcal{B}(\mathcal{H}), A \oplus B$ is the operator defined on $\mathcal{B}(\mathcal{H} \oplus \mathcal{H})$ by $\left(\begin{array}{cc}A & 0 \\ 0 & B\end{array}\right)$. A linear map $\Phi: \mathcal{B}(\mathcal{H}) \rightarrow \mathcal{B}(\mathcal{K})$ is positive if $\Phi(A) \geqslant 0$ whenever $A \geqslant 0$. It is said to be unital if $\Phi\left(\mathbf{1}_{\mathcal{H}}\right)=\mathbf{1}_{\mathcal{K}}$. A continuous function $f$ defined on the interval $J$ is called an operator convex function if $f((1-v) A+v B) \leqslant(1-v) f(A)+v f(B)$ for every $0<v<1$ and for every pair of bounded self-adjoint operators $A$ and $B$ whose spectra

(C) Petrozavodsk State University, 2019 
are both in $J$. For instance, the function $f(t)=t^{p}$ is operator convex on $(0, \infty)$ if either $1 \leqslant p \leqslant 2$ or $-1 \leqslant p \leqslant 0$.

The well known operator Jensen inequality (sometimes called the ChoiDavis-Jensen inequality) states:

$$
f(\Phi(A)) \leqslant \Phi(f(A)) .
$$

It holds for every operator convex $f: J \rightarrow \mathbb{R}$, self-adjoint operator $A$ with the spectra in $J$, and unital positive linear map $\Phi[2,3]$.

Hansen et al. [7] gave a general formulation of (1). The discrete version of their result reads as follows: If $f: J \rightarrow \mathbb{R}$ is an operator convex function, $A_{1}, \ldots, A_{n} \in \mathcal{B}(\mathcal{H})$ are self-adjoint operators with the spectra in $J$, and $\Phi_{1}, \ldots, \Phi_{n}: \mathcal{B}(\mathcal{H}) \rightarrow \mathcal{B}(\mathcal{K})$ are positive linear mappings such that $\sum_{i=1}^{n} \Phi_{i}\left(\mathbf{1}_{\mathcal{H}}\right)=\mathbf{1}_{\mathcal{K}}$, then

$$
f\left(\sum_{i=1}^{n} \Phi_{i}\left(A_{i}\right)\right) \leqslant \sum_{i=1}^{n} \Phi_{i}\left(f\left(A_{i}\right)\right) .
$$

Though, in the case of convex function inequality (2) does not hold in general (see [2, Remark 2.6]); we have the following estimate [4, Theorem 1]:

$$
f\left(\left\langle\sum_{i=1}^{n} \Phi_{i}\left(A_{i}\right) x, x\right\rangle\right) \leqslant\left\langle\sum_{i=1}^{n} \Phi_{i}\left(f\left(A_{i}\right)\right) x, x\right\rangle
$$

for any unit vector $x \in \mathcal{K}$. For recent results on the Jensen operator inequality, we refer the reader to [8-10].

Let $f: J \rightarrow \mathbb{R}$ be a convex function, $A \in \mathcal{B}(\mathcal{H})$ self-adjoint operator with spectrum in $J$, and let $x \in \mathcal{H}$ be a unit vector. Then, from [11],

$$
f(\langle A x, x\rangle) \leqslant\langle f(A) x, x\rangle .
$$

The Hölder-McCarthy inequality is a special case of (4):

$$
\langle A x, x\rangle^{p} \leqslant\left\langle A^{p} x, x\right\rangle,(x \in \mathcal{H} ;\|x\|=1),
$$

where $A$ is a positive operator and $p>1$. If the operator is positive and invertible, (5) is also true for $p<0$ (see, e.g., $[12,13]$ ).

Replace $A$ with $\Phi(A)$, where $\Phi: \mathcal{B}(\mathcal{H}) \rightarrow \mathcal{B}(\mathcal{K})$ is a unital positive linear map, we get

$$
f(\langle\Phi(A) x, x\rangle) \leqslant\langle f(\Phi(A)) x, x\rangle
$$


for any unit vector $x \in \mathcal{K}$. Assume that $A_{1}, \ldots, A_{n}$ are self-adjoint operators on $\mathcal{H}$ with the spectra in $J$ and $\Phi_{1}, \ldots, \Phi_{n}: \mathcal{B}(\mathcal{H}) \rightarrow \mathcal{B}(\mathcal{K})$ are positive linear maps with $\sum_{i=1}^{n} \Phi_{i}\left(\mathbf{1}_{\mathcal{H}}\right)=\mathbf{1}_{\mathcal{K}}$. Now apply inequality (6) to the self-adjoint operator $A$ on the Hilbert space $\mathcal{H} \oplus \cdots \oplus \mathcal{H}$ defined by $A=A_{1} \oplus \cdots \oplus A_{n}$ and the positive linear map $\Phi$ defined on $\mathcal{B}(\mathcal{H} \oplus \cdots \oplus \mathcal{H})$ by $\Phi(A)=\Phi_{1}\left(A_{1}\right) \oplus \cdots \oplus \Phi_{n}\left(A_{n}\right)$. Thus,

$$
f\left(\left\langle\sum_{i=1}^{n} \Phi_{i}\left(A_{i}\right) x, x\right\rangle\right) \leqslant\left\langle f\left(\sum_{i=1}^{n} \Phi_{i}\left(A_{i}\right)\right) x, x\right\rangle .
$$

This research paper is mainly focused on the inequalities of types (3) and (7), where two functions $f$ and $g$ are involved. This approach nicely extends the previously known results in the literature. The new inequalities are applied to obtain refinements of Hölder-McCarthy inequality (5). Additionally, we improve inequality (4) using the Cartesian decomposition of the operator.

2. Inequalities Related to Synchronous (Asynchronous) Functions. We say that functions $f, g: J \rightarrow \mathbb{R}$ are synchronous (asynchronous) on the interval $J$ if they satisfy the following condition:

$$
(f(t)-f(s))(g(t)-g(s)) \geqslant(\leqslant) 0
$$

for each $t, s \in J[1,6]$. For several recent results concerning synchronous (asynchronous) functions, see [5, 14].

The first result reads as follows.

Theorem 1. Let $f, g: J \rightarrow \mathbb{R}$ be continuous and synchronous functions, $A_{1}, \ldots, A_{n} \in \mathcal{B}(\mathcal{H})$ self-adjoint operators with the spectra in $J$, and let $\Phi_{1}, \ldots, \Phi_{n}: \mathcal{B}(\mathcal{H}) \rightarrow \mathcal{B}(\mathcal{K})$ be positive linear mappings such that $\sum_{i=1}^{n} \Phi_{i}\left(\mathbf{1}_{\mathcal{H}}\right)=\mathbf{1}_{\mathcal{K}}$. Then for any unit vector $x \in \mathcal{K}$,

$$
\begin{gathered}
\left\langle\sum_{i=1}^{n} \Phi_{i}\left(f\left(A_{i}\right) g\left(A_{i}\right)\right) x, x\right\rangle+f\left(\left\langle\sum_{i=1}^{n} \Phi_{i}\left(A_{i}\right) x, x\right\rangle\right) g\left(\left\langle\sum_{i=1}^{n} \Phi_{i}\left(A_{i}\right) x, x\right\rangle\right) \geqslant \\
\geqslant g\left(\left\langle\sum_{i=1}^{n} \Phi_{i}\left(A_{i}\right) x, x\right\rangle\right)\left\langle\sum_{i=1}^{n} \Phi_{i}\left(f\left(A_{i}\right)\right) x, x\right\rangle+ \\
+f\left(\left\langle\sum_{i=1}^{n} \Phi_{i}\left(A_{i}\right) x, x\right\rangle\right)\left\langle\sum_{i=1}^{n} \Phi_{i}\left(g\left(A_{i}\right)\right) x, x\right\rangle .
\end{gathered}
$$


The reverse inequality holds when $f, g$ are asynchronous functions.

Proof. We consider only the case of synchronous functions. It follows from (8) that

$$
f(t) g(t)+f(s) g(s) \geqslant f(t) g(s)+f(s) g(t)
$$

for each $t, s \in J$.

Fix $s \in J$. Since $J$ contains the spectra of the $A_{i}$ for $i=1, \ldots, n$, we may replace $t$ in the inequality (9) by $A_{i}$, via functional calculus to get

$$
f\left(A_{i}\right) g\left(A_{i}\right)+f(s) g(s) \mathbf{1}_{\mathcal{H}} \geqslant g(s) f\left(A_{i}\right)+f(s) g\left(A_{i}\right) .
$$

Applying the positive linear mappings $\Phi_{i}$ and summing on $i$ from 1 to $n$, this implies

$$
\begin{aligned}
& \sum_{i=1}^{n} \Phi_{i}\left(f\left(A_{i}\right) g\left(A_{i}\right)\right)+f(s) g(s) \mathbf{1}_{\mathcal{K}} \geqslant \\
& \geqslant g(s) \sum_{i=1}^{n} \Phi_{i}\left(f\left(A_{i}\right)\right)+f(s) \sum_{i=1}^{n} \Phi_{i}\left(g\left(A_{i}\right)\right) .
\end{aligned}
$$

The inequality (10) easily implies, for any $x \in \mathcal{K}$ with $\|x\|=1$,

$$
\begin{aligned}
& \left\langle\sum_{i=1}^{n} \Phi_{i}\left(f\left(A_{i}\right) g\left(A_{i}\right)\right) x, x\right\rangle+f(s) g(s) \geqslant \\
& \geqslant g(s)\left\langle\sum_{i=1}^{n} \Phi_{i}\left(f\left(A_{i}\right)\right) x, x\right\rangle+f(s)\left\langle\sum_{i=1}^{n} \Phi_{i}\left(g\left(A_{i}\right)\right) x, x\right\rangle .
\end{aligned}
$$

On the other hand, since $\sum_{i=1}^{n} \Phi_{i}\left(\mathbf{1}_{\mathcal{H}}\right)=\mathbf{1}_{\mathcal{K}}$ and the spectra of operators $A_{i}$, $i=1, \ldots, n$, are contained in the interval $J$, we have $\left\langle\sum_{i=1}^{n} \Phi_{i}\left(A_{i}\right) x, x\right\rangle \in J$, where $x \in \mathcal{K}$ with $\|x\|=1$. So, we may replace $s$ by $\left\langle\sum_{i=1}^{n} \Phi_{i}\left(A_{i}\right) x, x\right\rangle$ in (11). This yields

$$
\left\langle\sum_{i=1}^{n} \Phi_{i}\left(f\left(A_{i}\right) g\left(A_{i}\right)\right) x, x\right\rangle+f\left(\left\langle\sum_{i=1}^{n} \Phi_{i}\left(A_{i}\right) x, x\right\rangle\right) g\left(\left\langle\sum_{i=1}^{n} \Phi_{i}\left(A_{i}\right) x, x\right\rangle\right) \geqslant
$$




$$
\begin{aligned}
& \geqslant g\left(\left\langle\sum_{i=1}^{n} \Phi_{i}\left(A_{i}\right) x, x\right\rangle\right)\left\langle\sum_{i=1}^{n} \Phi_{i}\left(f\left(A_{i}\right)\right) x, x\right\rangle+ \\
& +f\left(\left\langle\sum_{i=1}^{n} \Phi_{i}\left(A_{i}\right) x, x\right\rangle\right)\left\langle\sum_{i=1}^{n} \Phi_{i}\left(g\left(A_{i}\right)\right) x, x\right\rangle .
\end{aligned}
$$

This completes the proof.

Remark. Let $A_{1}, \ldots, A_{n} \in \mathcal{B}(\mathcal{H})$ be positive operators, and let $\Phi_{1}, \ldots, \Phi_{n}$ : $\mathcal{B}(\mathcal{H}) \rightarrow \mathcal{B}(\mathcal{K})$ be positive linear mappings such that $\sum_{i=1}^{n} \Phi_{i}\left(\mathbf{1}_{\mathcal{H}}\right)=\mathbf{1}_{\mathcal{K}}$. Then, for any $p, q>0$,

$$
\begin{aligned}
& \left\langle\sum_{i=1}^{n} \Phi_{i}\left(A_{i}^{p+q}\right) x, x\right\rangle+\left\langle\sum_{i=1}^{n} \Phi_{i}\left(A_{i}\right) x, x\right\rangle^{p+q} \geqslant \\
& \geqslant\left\langle\sum_{i=1}^{n} \Phi_{i}\left(A_{i}\right) x, x\right\rangle^{q}\left\langle\sum_{i=1}^{n} \Phi_{i}\left(A_{i}^{p}\right) x, x\right\rangle+ \\
& +\left\langle\sum_{i=1}^{n} \Phi_{i}\left(A_{i}\right) x, x\right\rangle^{p}\left\langle\sum_{i=1}^{n} \Phi_{i}\left(A_{i}^{q}\right) x, x\right\rangle
\end{aligned}
$$

for any unit vector $x \in \mathcal{K}$. If $A_{1}, \ldots, A_{n} \in \mathcal{B}(\mathcal{H})$ are positive invertible operators, then (12) also holds for $p, q<0$.

If $A_{1} \ldots, A_{n} \in \mathcal{B}(\mathcal{H})$ are positive invertible and either $p>0, q<0$ or $p<0, q>0$, then the reverse inequality holds in (12).

Corollary. Let $f, g: J \rightarrow \mathbb{R}$ be synchronous functions, $A_{1}, \ldots, A_{n} \in \mathcal{B}(\mathcal{H})$ self-adjoint operators with the spectra in $J$, and let $w_{1}, \ldots, w_{n}$ be positive scalars such that $\sum_{i=1}^{n} w_{i}=1$. Then, for any unit vector $x \in \mathcal{H}$,

$$
\begin{aligned}
\left\langle\sum_{i=1}^{n} w_{i} f\left(A_{i}\right)\right. & \left.g\left(A_{i}\right) x, x\right\rangle+f\left(\left\langle\sum_{i=1}^{n} w_{i} A_{i} x, x\right\rangle\right) g\left(\left\langle\sum_{i=1}^{n} w_{i} A_{i} x, x\right\rangle\right) \geqslant \\
\geqslant & g\left(\left\langle\sum_{i=1}^{n} w_{i} A_{i} x, x\right\rangle\right)\left\langle\sum_{i=1}^{n} w_{i} f\left(A_{i}\right) x, x\right\rangle+ \\
& +f\left(\left\langle\sum_{i=1}^{n} w_{i} A_{i} x, x\right\rangle\right)\left\langle\sum_{i=1}^{n} w_{i} g\left(A_{i}\right) x, x\right\rangle .
\end{aligned}
$$

The reverse inequality holds when $f, g$ are asynchronous functions. 
Proof. Apply Theorem 1 for positive linear mappings $\Phi_{i}: \mathcal{B}(\mathcal{H}) \rightarrow \mathcal{B}(\mathcal{H})$ determined by $\Phi_{i}: T \mapsto w_{i} T(i=1, \ldots, n)$.

Remark. Suppose, in addition to the assumptions in Theorem 1, that $f$ is convex on $J$; then

$$
\begin{aligned}
& g\left(\left\langle\sum_{i=1}^{n} \Phi_{i}\left(A_{i}\right) x, x\right\rangle\right)\left\langle\sum_{i=1}^{n} \Phi_{i}\left(f\left(A_{i}\right)\right) x, x\right\rangle+ \\
& +f\left(\left\langle\sum_{i=1}^{n} \Phi_{i}\left(A_{i}\right) x, x\right\rangle\right)\left\langle\sum_{i=1}^{n} \Phi_{i}\left(g\left(A_{i}\right)\right) x, x\right\rangle \leqslant \\
& \leqslant\left\langle\sum_{i=1}^{n} \Phi_{i}\left(f\left(A_{i}\right) g\left(A_{i}\right)\right) x, x\right\rangle+f\left(\left\langle\sum_{i=1}^{n} \Phi_{i}\left(A_{i}\right) x, x\right\rangle\right) g\left(\left\langle\sum_{i=1}^{n} \Phi_{i}\left(A_{i}\right) x, x\right\rangle\right) \leqslant \\
& \leqslant\left\langle\sum_{i=1}^{n} \Phi_{i}\left(f\left(A_{i}\right) g\left(A_{i}\right)\right) x, x\right\rangle+\left\langle\sum_{i=1}^{n} \Phi_{i}\left(f\left(A_{i}\right)\right) x, x\right\rangle g\left(\left\langle\sum_{i=1}^{n} \Phi_{i}\left(A_{i}\right) x, x\right\rangle\right)
\end{aligned}
$$

for any unit vector $x \in \mathcal{K}$, due to (3). Therefore,

$$
\begin{aligned}
& f\left(\left\langle\sum_{i=1}^{n} \Phi_{i}\left(A_{i}\right) x, x\right\rangle\right)\left\langle\sum_{i=1}^{n} \Phi_{i}\left(g\left(A_{i}\right)\right) x, x\right\rangle \leqslant \\
& \leqslant\left\langle\sum_{i=1}^{n} \Phi_{i}\left(f\left(A_{i}\right) g\left(A_{i}\right)\right) x, x\right\rangle+ \\
&+g\left(\left\langle\sum_{i=1}^{n} \Phi_{i}\left(A_{i}\right) x, x\right\rangle\right)\left[f\left(\left\langle\sum_{i=1}^{n} \Phi_{i}\left(A_{i}\right) x, x\right\rangle\right)-\left\langle\sum_{i=1}^{n} \Phi_{i}\left(f\left(A_{i}\right)\right) x, x\right\rangle\right] \leqslant \\
& \leqslant\left\langle\sum_{i=1}^{n} \Phi_{i}\left(f\left(A_{i}\right) g\left(A_{i}\right)\right) x, x\right\rangle .
\end{aligned}
$$

If, in addition, $g$ is convex on $J$, then

$$
\begin{aligned}
& f\left(\left\langle\sum_{i=1}^{n} \Phi_{i}\left(A_{i}\right) x, x\right\rangle\right) g\left(\left\langle\sum_{i=1}^{n} \Phi_{i}\left(A_{i}\right) x, x\right\rangle\right) \leqslant \\
& \leqslant f\left(\left\langle\sum_{i=1}^{n} \Phi_{i}\left(A_{i}\right) x, x\right\rangle\right)\left\langle\sum_{i=1}^{n} \Phi_{i}\left(g\left(A_{i}\right)\right) x, x\right\rangle \leqslant\left\langle\sum_{i=1}^{n} \Phi_{i}\left(f\left(A_{i}\right) g\left(A_{i}\right)\right) x, x\right\rangle+ \\
& +g\left(\left\langle\sum_{i=1}^{n} \Phi_{i}\left(A_{i}\right) x, x\right\rangle\right)\left[f\left(\left\langle\sum_{i=1}^{n} \Phi_{i}\left(A_{i}\right) x, x\right\rangle\right)-\left\langle\sum_{i=1}^{n} \Phi_{i}\left(f\left(A_{i}\right)\right) x, x\right\rangle\right] \leqslant
\end{aligned}
$$




$$
\leqslant\left\langle\sum_{i=1}^{n} \Phi_{i}\left(f\left(A_{i}\right) g\left(A_{i}\right)\right) x, x\right\rangle
$$

holds for any unit vector $x \in \mathcal{K}$.

As a direct consequence of (13), we have:

Corollary. Let $A_{1}, \ldots, A_{n} \in \mathcal{B}(\mathcal{H})$ be positive operators, and let $\Phi_{1}, \ldots, \Phi_{n}: \mathcal{B}(\mathcal{H}) \rightarrow \mathcal{B}(\mathcal{K})$ be positive linear mappings, such that $\sum_{i=1}^{n} \Phi_{i}\left(\mathbf{1}_{\mathcal{H}}\right)=\mathbf{1}_{\mathcal{K}}$. Then, for any $p, q>1$,

$$
\begin{aligned}
&\left\langle\sum_{i=1}^{n} \Phi_{i}\left(A_{i}\right) x, x\right\rangle^{p+q} \leqslant\left\langle\sum_{i=1}^{n} \Phi_{i}\left(A_{i}\right) x, x\right\rangle^{p}\left\langle\sum_{i=1}^{n} \Phi_{i}\left(A_{i}^{q}\right) x, x\right\rangle \leqslant \\
& \leqslant\left\langle\sum_{i=1}^{n} \Phi_{i}\left(A_{i}^{p+q}\right) x, x\right\rangle+ \\
&+\left\langle\sum_{i=1}^{n} \Phi_{i}\left(A_{i}\right) x, x\right\rangle^{q}\left[\left\langle\sum_{i=1}^{n} \Phi_{i}\left(A_{i}\right) x, x\right\rangle^{p}-\left\langle\sum_{i=1}^{n} \Phi_{i}\left(A_{i}^{p}\right) x, x\right\rangle\right] \leqslant \\
& \leqslant\left\langle\sum_{i=1}^{n} \Phi_{i}\left(A_{i}^{p+q}\right) x, x\right\rangle
\end{aligned}
$$

for any unit vector $x \in \mathcal{K}$.

If $A_{1}, \ldots, A_{n} \in \mathcal{B}(\mathcal{H})$ are positive invertible operators, then (14) also holds for $p, q<0$.

The second main result reads as follows:

Theorem 2. Let $f, g: J \rightarrow \mathbb{R}$ be synchronous functions, $A_{1}, \ldots, A_{n} \in \mathcal{B}(\mathcal{H})$ self-adjoint operators with the spectra in $J$, and let $\Phi_{1}, \ldots, \Phi_{n}$ : $\mathcal{B}(\mathcal{H}) \rightarrow \mathcal{B}(\mathcal{K})$ be positive linear mappings such that $\sum_{i=1}^{n} \Phi_{i}\left(\mathbf{1}_{\mathcal{H}}\right)=\mathbf{1}_{\mathcal{K}}$. Then, for any unit vector $x \in \mathcal{K}$,

$$
\begin{aligned}
\left\langle f\left(\sum_{i=1}^{n} \Phi_{i}\left(A_{i}\right)\right) g\left(\sum_{i=1}^{n} \Phi_{i}\left(A_{i}\right)\right) x, x\right\rangle+ \\
+f\left(\left\langle\sum_{i=1}^{n} \Phi_{i}\left(A_{i}\right) x, x\right\rangle\right) g\left(\left\langle\sum_{i=1}^{n} \Phi_{i}\left(A_{i}\right) x, x\right\rangle\right) \geqslant
\end{aligned}
$$




$$
\begin{aligned}
\geqslant g\left(\left\langle\sum_{i=1}^{n} \Phi_{i}\left(A_{i}\right) x, x\right\rangle\right)\left\langle f\left(\sum_{i=1}^{n} \Phi_{i}\left(A_{i}\right)\right) x, x\right\rangle+ \\
\quad+f\left(\left\langle\sum_{i=1}^{n} \Phi_{i}\left(A_{i}\right) x, x\right\rangle\right)\left\langle g\left(\sum_{i=1}^{n} \Phi_{i}\left(A_{i}\right)\right) x, x\right\rangle .
\end{aligned}
$$

The reverse inequality holds when $f, g$ are asynchronous functions.

Proof. Fix $s \in J$. Since $J$ contains the spectra of the $A_{i}$ for $i=1, \ldots, n$ and $\sum_{i=1}^{n} \Phi_{i}\left(\mathbf{1}_{\mathcal{H}}\right)=\mathbf{1}_{\mathcal{K}}$, the spectra of $\sum_{i=1}^{n} \Phi_{i}\left(A_{i}\right)$ are also contained in $J$. Then, we may replace $t$ in the inequality (9) by $\sum_{i=1}^{n} \Phi_{i}\left(A_{i}\right)$, via functional calculus to get

$$
\begin{aligned}
f\left(\sum_{i=1}^{n} \Phi_{i}\left(A_{i}\right)\right) g\left(\sum_{i=1}^{n} \Phi_{i}\left(A_{i}\right)\right)+f(s) g(s) \mathbf{1}_{\mathcal{K}} \geqslant & \\
& \geqslant g(s) f\left(\sum_{i=1}^{n} \Phi_{i}\left(A_{i}\right)\right)+f(s) g\left(\sum_{i=1}^{n} \Phi_{i}\left(A_{i}\right)\right) .
\end{aligned}
$$

This inequality implies, for any $x \in \mathcal{K}$ with $\|x\|=1$,

$$
\begin{aligned}
& \left\langle f\left(\sum_{i=1}^{n} \Phi_{i}\left(A_{i}\right)\right) g\left(\sum_{i=1}^{n} \Phi_{i}\left(A_{i}\right)\right) x, x\right\rangle+f(s) g(s) \geqslant \\
& \geqslant g(s)\left\langle f\left(\sum_{i=1}^{n} \Phi_{i}\left(A_{i}\right)\right) x, x\right\rangle+f(s)\left\langle g\left(\sum_{i=1}^{n} \Phi_{i}\left(A_{i}\right)\right) x, x\right\rangle .
\end{aligned}
$$

Substituting $s$ with $\left\langle\sum_{i=1}^{n} \Phi_{i}\left(A_{i}\right) x, x\right\rangle$ in (15), gives the desired result.

Remark. Suppose, in addition to the assumptions in Theorem 2, that $f$ is convex on $J$; then

$$
\begin{aligned}
& f\left(\left\langle\sum_{i=1}^{n} \Phi_{i}\left(A_{i}\right) x, x\right\rangle\right)\left\langle g\left(\sum_{i=1}^{n} \Phi_{i}\left(A_{i}\right)\right) x, x\right\rangle \\
& \leqslant\left\langle f\left(\sum_{i=1}^{n} \Phi_{i}\left(A_{i}\right)\right) g\left(\sum_{i=1}^{n} \Phi_{i}\left(A_{i}\right)\right) x, x\right\rangle+g\left(\left\langle\sum_{i=1}^{n} \Phi_{i}\left(A_{i}\right) x, x\right\rangle\right) \times \\
& \times\left[f\left(\left\langle\sum_{i=1}^{n} \Phi_{i}\left(A_{i}\right) x, x\right\rangle\right)-\left\langle f\left(\sum_{i=1}^{n} \Phi_{i}\left(A_{i}\right)\right) x, x\right\rangle\right] \leqslant
\end{aligned}
$$




$$
\leqslant\left\langle f\left(\sum_{i=1}^{n} \Phi_{i}\left(A_{i}\right)\right) g\left(\sum_{i=1}^{n} \Phi_{i}\left(A_{i}\right)\right) x, x\right\rangle
$$

for any unit vector $x \in \mathcal{K}$, due to (7). If, in addition, $g$ is convex on $J$,

$$
\begin{gathered}
f\left(\left\langle\sum_{i=1}^{n} \Phi_{i}\left(A_{i}\right) x, x\right\rangle\right) g\left(\left\langle\sum_{i=1}^{n} \Phi_{i}\left(A_{i}\right) x, x\right\rangle\right) \leqslant \\
\leqslant f\left(\left\langle\sum_{i=1}^{n} \Phi_{i}\left(A_{i}\right) x, x\right\rangle\right)\left\langle g\left(\sum_{i=1}^{n} \Phi_{i}\left(A_{i}\right)\right) x, x\right\rangle \leqslant \\
\leqslant\left\langle f\left(\sum_{i=1}^{n} \Phi_{i}\left(A_{i}\right)\right) g\left(\sum_{i=1}^{n} \Phi_{i}\left(A_{i}\right)\right) x, x\right\rangle+g\left(\left\langle\sum_{i=1}^{n} \Phi_{i}\left(A_{i}\right) x, x\right\rangle\right) \times \\
\times\left[f\left(\left\langle\sum_{i=1}^{n} \Phi_{i}\left(A_{i}\right) x, x\right\rangle\right)-\left\langle f\left(\sum_{i=1}^{n} \Phi_{i}\left(A_{i}\right)\right) x, x\right\rangle\right] \leqslant \\
\leqslant\left\langle f\left(\sum_{i=1}^{n} \Phi_{i}\left(A_{i}\right)\right) g\left(\sum_{i=1}^{n} \Phi_{i}\left(A_{i}\right)\right) x, x\right\rangle
\end{gathered}
$$

holds for any unit vector $x \in \mathcal{K}$.

3. Refinement via the Cartesian Decomposition. We start this section by proving the following theorem, that can be considered as a refinement of $[4$, Theorem 1$]$.

Theorem 3. Let $\Phi: \mathcal{B}(\mathcal{H}) \rightarrow \mathcal{B}(\mathcal{K})$ be a unital positive linear map, $A \in \mathcal{B}(\mathcal{H})$ be a positive operator with the Cartesian decomposition $A=B+i C$, and let $f$ be a non-negative function on $[0, \infty)$, such that $g(t)=f(\sqrt{t})$ is convex. Then, for any unit vector $x \in \mathcal{K}$,

$f(\langle\Phi(A) x, x\rangle) \leqslant\left\{\begin{array}{l}f\left(\left(\left\langle\Phi(B)^{2} x, x\right\rangle+\langle\Phi(C) x, x\rangle^{2}\right)^{\frac{1}{2}}\right) \\ f\left(\left(\langle\Phi(B) x, x\rangle^{2}+\left\langle\Phi(C)^{2} x, x\right\rangle\right)^{\frac{1}{2}}\right)\end{array} \leqslant\langle\Phi(f(A)) x, x\rangle\right.$.

Proof. First of all, note that

$$
\langle\Phi(A) x, x\rangle^{2}=\langle\Phi(B) x, x\rangle^{2}+\langle\Phi(C) x, x\rangle^{2},(x \in \mathcal{K} ;\|x\|=1)
$$

and

$$
\Phi(A)^{2}=\Phi(B)^{2}+\Phi(C)^{2}
$$


Since $g$ is non-negative and convex on $[0, \infty)$, it follows that $g$ is increasing. Now,

$$
\begin{aligned}
& g\left(\langle\Phi(A) x, x\rangle^{2}\right)=g\left(\langle\Phi(B) x, x\rangle^{2}+\langle\Phi(C) x, x\rangle^{2}\right) \leqslant(\text { by }(16)) \\
& \leqslant g\left(\left\langle\Phi(B)^{2} x, x\right\rangle+\langle\Phi(C) x, x\rangle^{2}\right) \leqslant(\text { by the Cauchy-Schwarz inequality) } \\
& \leqslant g\left(\left\langle\Phi(B)^{2}+\Phi(C)^{2} x, x\right\rangle\right)=(\text { by }(5)) \\
& =g\left(\left\langle\Phi(A)^{2} x, x\right\rangle\right) \leqslant(\text { by }(17)) \\
& \leqslant g\left(\left\langle\Phi\left(A^{2}\right) x, x\right\rangle\right) \leqslant\left(\text { since } t^{2} \text { is operator convex }\right) \\
& \leqslant\left\langle\Phi\left(g\left(A^{2}\right)\right) x, x\right\rangle \quad(\text { by }(3))
\end{aligned}
$$

for any unit vector $x \in \mathcal{K}$. Consequently,

$$
\begin{aligned}
f(\langle\Phi(A) x, x\rangle) & =g\left(\langle\Phi(A) x, x\rangle^{2}\right) \leqslant \\
& \leqslant g\left(\left\langle\Phi(B)^{2} x, x\right\rangle+\langle\Phi(C) x, x\rangle^{2}\right)= \\
& =f\left(\left(\left\langle\Phi(B)^{2} x, x\right\rangle+\langle\Phi(C) x, x\rangle^{2}\right)^{\frac{1}{2}}\right) \leqslant \\
& \leqslant\left\langle\Phi\left(g\left(A^{2}\right)\right) x, x\right\rangle= \\
& =\langle\Phi(f(A)) x, x\rangle .
\end{aligned}
$$

The other case can be obtained similarly.

By setting $f(t)=t^{p}(t \geqslant 0, p \geqslant 2)$ in Theorem 3 , we find that:

Corollary. Let $\Phi: \mathcal{B}(\mathcal{H}) \rightarrow \mathcal{B}(\mathcal{K})$ be a unital positive linear map, $A \in \mathcal{B}(\mathcal{H})$ be a positive operator with the Cartesian decomposition $A=B+i C$. Then, for any $p \geqslant 2$,

$$
\langle\Phi(A) x, x\rangle^{p} \leqslant\left\{\begin{array}{l}
\left(\left\langle\Phi(B)^{2} x, x\right\rangle+\langle\Phi(C) x, x\rangle^{2}\right)^{\frac{p}{2}} \\
\left(\langle\Phi(B) x, x\rangle^{2}+\left\langle\Phi(C)^{2} x, x\right\rangle\right)^{\frac{p}{2}} \leqslant\left\langle\Phi\left(A^{p}\right) x, x\right\rangle
\end{array}\right.
$$

for any unit vector $x \in \mathcal{K}$.

Taking $\Phi(T)=T$ in Theorem 3, we get:

Corollary. Let $A \in \mathcal{B}(\mathcal{H})$ be a positive operator with the Cartesian decomposition $A=B+i C$, and let $f$ be a non-negative function on 
$[0, \infty)$, such that $g(t)=f(\sqrt{t})$ is convex. Then, for any unit vector $x \in \mathcal{H}$,

$$
f(\langle A x, x\rangle) \leqslant\left\{\begin{array}{l}
f\left(\left(\left\langle B^{2} x, x\right\rangle+\langle C x, x\rangle^{2}\right)^{\frac{1}{2}}\right) \\
f\left(\left(\langle B x, x\rangle^{2}+\left\langle C^{2} x, x\right\rangle\right)^{\frac{1}{2}}\right)
\end{array} \leqslant\langle f(A) x, x\rangle .\right.
$$

In particular, for any $p \geqslant 2$,

$$
\langle A x, x\rangle^{p} \leqslant\left\{\begin{array}{l}
\left(\left\langle B^{2} x, x\right\rangle+\langle C x, x\rangle^{2}\right)^{\frac{p}{2}} \\
\left(\langle B x, x\rangle^{2}+\left\langle C^{2} x, x\right\rangle\right)^{\frac{p}{2}} \leqslant\left\langle A^{p} x, x\right\rangle
\end{array}\right.
$$

holds for any unit vector $x \in \mathcal{H}$.

Acknowledgment. The authors would like to express their hearty thanks to the referees for their valuable comments.

This work was financially supported by Safadasht Branch, Islamic Azad University, Tehran, Iran.

\section{References}

[1] Bourin J.-C. Some inequalities for norms on matrices and operators, Linear Algebra Appl., 1999, vol. 292, pp. 139-154.

DOI: https://doi.org/10.1016/S0024-3795(99)00024-5

[2] Choi M. D., A Schwarz inequality for positive linear maps on $C^{*}$-algebras, Illinois J. Math., 1974, vol. 18, no. 4, pp. 565-574.

DOI: https://doi.org/10.1215/ijm/1256051007

[3] Davis C. A Schwarz inequality for convex operator functions, Proc. Amer. Math. Soc., 1957, vol. 8, pp. 42-44.

DOI: https://doi.org/10.1090/S0002-9939-1957-0084120-4.

[4] Dragomir S. S. A weakened version of Davis-Choi-Jensen's inequality for normalised positive linear maps, Proyecciones., 2017, vol. 36, no. 1, pp. 81-94.

DOI: http://doi.org/10.4067/S0716-09172017000100005

[5] Dragomir S. S. Čebyšev's type inequalities for functions of selfadjoint operators in Hilbert spaces, Linear Multilinear Algebra., 2010, vol. 58, no. 7, pp. $805-814$.

DOI: https://doi.org/10.1080/03081080902992104 
[6] Fujii J.-I. A trace inequality arising from quantum information theory, Linear Algebra Appl., 2005, vol. 400, pp. 141-146.

DOI: https://doi.org/10.1016/j.laa.2004.11.009

[7] Hansen F., Pečarić J., Perić I. Jensen's operator inequality and it's converses, Math. Scand., 2007, vol. 100, no. 1, pp. 61-73.

DOI: https://doi.org/10.7146/math.scand.a-15016

[8] Horváth L., Khan K. A., Pečarić J. Cyclic refinements of the different versions of operator Jensen's inequality, Electron. J. Linear Algebra., 2016, vol. 31 , pp. $125-133$.

DOI: https://doi.org/10.13001/1081-3810.3098

[9] Mićić J., Moradi H. R., Furuichi S. Choi-Davis-Jensen's inequality without convexity, J. Math. Inequal., 2018, vol. 12, no. 4, pp. 1075-1085.

DOI: https://doi.org/10.7153/jmi-2018-12-82

[10] Mićić J., Pečarić J. Some mappings related to Levinson's inequality for Hilbert space operators, Filomat., 2017, vol. 31, no. 7, pp. 1995-2009.

DOI: https://doi.org/10.2298/FIL1707995M

[11] Mond B., Pečarić J. On Jensen's inequality for operator convex functions, Houston J. Math., 1995, vol. 21, pp. 739-754.

[12] Moradi H. R., Furuichi S., Mitroi F. C., Naseri R. An extension of Jensen's operator inequality and its application to Young inequality, Rev. R. Acad. Cienc. Exactas Fís. Nat. Ser. A Mat., 2019, vol. 113, no. 2, pp. 605-614. DOI: https://doi.org/10.1007/s13398-018-0499-7

[13] Moradi H. R., Omidvar M. E., Adil Khan M., Nikodem K. Around Jensen's inequality for strongly convex functions, Aequat. Math., 2018, vol. 92, no. 1, pp. 25-37.

DOI: https://doi.org/10.1007/s00010-017-0496-5

[14] Moradi H. R., Omidvar M. E., Dragomir S. S. An operator extension of Čebyšev inequality, An. Ştiinţ. Univ. "Ovidius" Constanţa Ser. Mat., 2017, vol. 25 , no. 2, pp. $135-147$.

DOI: https://doi.org/10.1515/auom-2017-0025

Received May 28, 2019.

In revised form, July 18, 2019.

Accepted July 21, 2019.

Published online August 14, 2019.

B. Moosavi

Department of Mathematics, Safadasht Branch, Islamic Azad University, Tehran, Iran.

E-mail: baharak_moosavie@yahoo.com 
H. R. Moradi

Young Researchers and Elite Club, Mashhad Branch, Islamic Azad University, Mashhad, Iran.

E-mail: hrmoradi@mshdiau.ac.ir

\section{Shah Hosseini}

Department of Mathematics, Shahr-e-Qods Branch, Islamic Azad University, Tehran, Iran.

E-mail: mohsen_shahhosseini@yahoo.com 